\title{
Local Buckling of Concrete Filled Rectangular Steel Tube with Longitudinal Stiffener under Axial Compression
}

\author{
Hongping LIU*, Zhiheng ZHANG, Zhang CHENG, Jin WEI, Guoliang ZENG
}

\begin{abstract}
Width-thickness ratio was an important parameter for designing Concrete Filled Rectangular Steel Tube (CFRST). Welding longitudinal stiffener on the internal wall of steel pipe could delay the local buckling, which increased the limit of width-thickness ratio. If there was not enough stiffener and its sectional dimension was too small, the local buckling of steel pipe would occur, inducing its bearing capacity seriously. If the stiffener sectional dimension was too large, concrete filled in steel tube would be broken up, which reduces its bearing capacity. To solve that problem, this paper studied local buckling of CFRST with longitudinal stiffener under axial compression and design of longitudinal stiffener. It established buckling analysis model, simplified local buckling analysis as calculating buckling load of thin plate clamped on loading side and unloading side under axial force. It deduced buckling load and buckling coefficient based on the principle of energy. The results showed that buckling mode depended on stiffening rigidity. Therefore, it put forward minimum stiffening rigidity ratio that controlled the stiffener design. This paper also came up with a formula to calculate minimum stiffening rigidity ratio. It provided guidance on designing number, sectional dimension and material performance.
\end{abstract}

Keywords: analytical solution; concrete-filled steel tube; design; local buckling; longitudinal stiffener; rectangular

\section{INTRODUCTION}

Concrete filled steel tube (CFST) is widely used to be chord member of truss bridges, arch rib of arch bridges, towers and pillars of cable-stayed bridges owing to numerous advantages, such as high bearing capacity, good plasticity and toughness of material, convenient construction, good fire resistance and economic benefits, becoming a structure form having bright future [1]. Compared with circular cross sections, Concrete Filled Rectangular Steel Tubes (CFRST) have dominant positions, such as larger member sizes, relatively easier connection structures, and more convenient constructions, in high width-thickness ratio structures. Width-thickness ratio of steel cubes is a main parameter restricting design of CFRST cross sections [2]. Too excessive values of width-thickness ratio can lead to the occurrence of out of plane buckling before yielding failure of steel plates. However, too small values also cause some difficulties, such as limited sizes of member cross sections, huge steel consumption. In order to increase limited values of widththickness ratio and delay local buckling of tube wall, longitudinal stiffeners can be welded on the internal wall of steel pipe [3]. The sizes of longitudinal stiffeners will increase as the ratio becomes larger. For some large cross sections of CFRST, the height of stiffeners should be improved, constraining the overall performance of core concrete and bringing trouble to construction.

CFRST columns would locally buckle in tube walls under the load of axial compression. As a result, core concrete of cubes can be considered as rigid material. What is more, deformed functions corresponding with boundary conditions can also be assumed. In this way, energy approach can be applied to derive the formula about steel cube local buckling intensity; limited values of buckling coefficient and width-thickness ratio in distinct boundary conditions are determined as well [3]. He Baokang et.al [4, 5] simplified non-loading edges of steel plates to the fixed boundary, deriving a conclusion that the local buckling coefficient of CFRST is 2.67 times bigger than steel hollow pipes. Mo Shixu et.al [6] further considered non-loading edges of steel plates as elastic constraints, determining the relation equation between constraint stiffness and local buckling intensity of CFRST columns. Cai Jian et al. [7, 8] derived the local buckling coefficients of CFRST with binding bars under the axial compression by the same approach, analyzing the influence of cube steel widththickness ratio limited values, which exerts constraint tension trussing parameters. Cheng Rong et al. [9] analyzed variation parameters of buckling coefficients about CFRST dwarf columns with longitudinal stiffeners under axial compression, discussing the influence of steel cubes' stability when dimensions of stiffeners vary. Based on these researches, this paper applies energy method to derive computing formula about steel cube wall local buckling intensity of CFRST with longitudinal stiffeners under axial compression, discussing several important parameters such as the amount of stiffeners, cross section sizes and material performance, which exert influence to cube local buckling performance, determining a computing formula of minimal stiffener rigid ratio about external cube steel with total stiffeners, putting forward structure requirements of stiffeners and design determining formula.

\section{LOCAL BUCKLING MODEL ESTABLISHMENT OF CFRST COLUMNS WITH LONGITUDINAL STIFFENERS UNDER AXIAL COMPRESSION}

As for CFRST columns with longitudinal stiffeners under axial compression, concrete in tubes can be considered as rigid material. Steel cube walls can only be convexly deformed due to the supporting effect of the concrete when it locally buckles. What's more, the lengths of plates are relatively much bigger than the widths. As a result, transverse could be a semi-wave and in longitudinal direction plates could be a series of continuous semi-waves when steel plates locally buckle, as shown in Fig. 1.

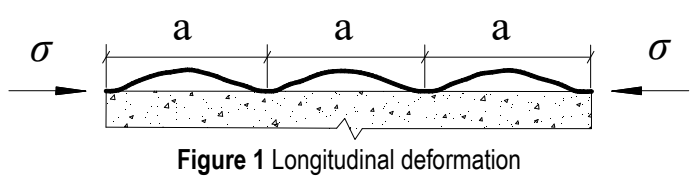


Steel plates can only be buckling outward between stiffeners. In addition, in the corner of steel cubes sustain right angles all the time. That is to say, each side of plates cannot rotate, which means none-loaded sides of plates are fixed sides. Based on the shapes of longitudinal deformation curves and curves' continuity, the sides whose first derivative equals zero may emerge in the transition process from one convex part to another. Plates whose two sides' first derivatives are zero can be served as fixed sides. It will be seen from these that the analysis of local buckling CFRST columns with longitudinal stiffeners under axial compression can be simplified to solving plates' buckling load under axial compression. And all the boundary conditions are clamped boundary sheets, which can only buckle from one side.

As for CFRST columns with longitudinal stiffeners under axial compression, if flexural stiffness is large enough, longitudinal ribs can offer supporting edges for plates and the buckling pattern will be shown as Fig. 2b. if stiffeners can stay straight all the time. Otherwise, stiffeners can bulge together with plates, only served as elastic bearing. And the pattern has much similarity with CFRST without stiffeners as shown in Fig. 2 a.

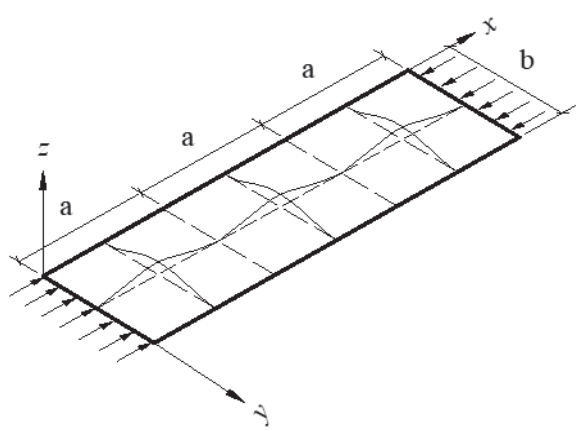

(a) Incomplete stiffening

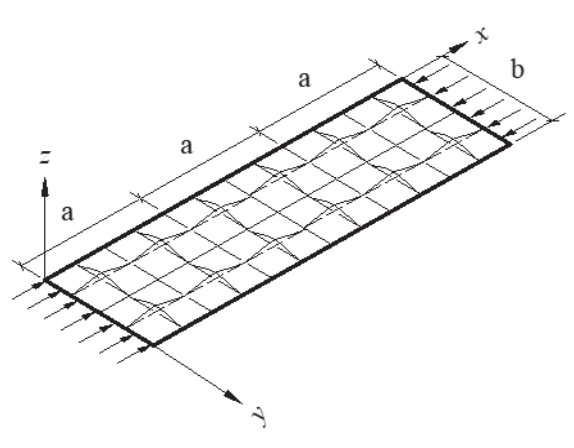

(b) Complete stiffening

Figure 2 Buckling deformation

Based on these analyses, buckling analysis of CFRST columns with longitudinal stiffeners under axial compression can be simplified to solving buckling load of thin plates under distributed loads. And all the boundary conditions are clamped boundary sheets, which can only buckle from one side.

\section{USING ENERGY APPROACH TO SOLVE BUCKLING LOAD OF CFRST COLUMNS WITH LONGITUDINAL STIFFENERS UNDER AXIAL COMPRESSION}

Strain energy of small deformation about rectangular thin-wall steel tubes can be shown as:

$$
U_{\mathrm{p}}=\frac{D}{2} \int_{0}^{b} \int_{0}^{a}\left\{\begin{array}{l}
{\left[\frac{\partial^{2} w}{\partial x^{2}}+\frac{\partial^{2} w}{\partial y^{2}}\right]^{2}} \\
+2(1-v)\left[\left(\frac{\partial^{2} w}{\partial x \partial y}\right)^{2}-\frac{\partial^{2} w}{\partial x^{2}} \frac{\partial^{2} w}{\partial y^{2}}\right]
\end{array}\right\} \mathrm{d} x \mathrm{~d} y
$$

In Eq. (1), $a$ is length of plates, $b$ is width of plates, $w$ is deflection function, $D=\frac{E t^{3}}{12\left(1-v^{2}\right)}$ is flexural rigidity of per unit wide plates. In this formula, $t$ is thickness of steel plates, $E$ is elasticity modulus of plates, $v$ is Poisson's ratio of steel plates.

External potential energy of steel plates under unidirectional uniform axial compression stress $\sigma$ is:

$V_{\mathrm{p}}=-\frac{1}{2} \int_{0}^{b} \int_{0}^{a} \sigma b\left(\frac{\partial w}{\partial x}\right)^{2} \mathrm{~d} x \mathrm{~d} y$

Steel tubes local buckling performance under axial compression can be influenced by longitudinal stiffeners. As a result, the rigidity of stiffeners should be analyzed. When steel plates buckle under unidirectional and uniform axial compression, longitudinal stiffeners can be thought to deform together with steel plates. As a consequence, bend strain energy $U_{\mathrm{s}}$ and external potential energy $V_{\mathrm{s}}$ can be shown as follows:

$U_{\mathrm{s}}=\frac{E I_{\mathrm{s}}}{2} \sum_{i=1}^{n_{\mathrm{s}}} \int_{0}^{a}\left(\frac{\partial^{2} w}{\partial x^{2}}\right)_{y=i \cdot b /\left(n_{\mathrm{s}}+1\right)}^{2} \mathrm{~d} x$

$V_{\mathrm{s}}=-\frac{1}{2} \sum_{i=1}^{n_{\mathrm{S}}} \int_{0}^{a} \sigma_{x} b_{\mathrm{s}} t_{\mathrm{s}}\left(\frac{\partial w}{\partial x}\right)_{y=i \cdot b /\left(n_{\mathrm{S}}+1\right)}^{2} \mathrm{~d} x$

In Eq. (4), $b_{\mathrm{s}}$ and $t_{\mathrm{s}}$ are width and thickness of stiffeners respectively, $I_{\mathrm{s}}$ is moment of inertia of neutral axis cross section of stiffeners and $I_{\mathrm{s}}=\frac{1}{12} t_{\mathrm{s}} b_{\mathrm{s}}^{3} \cdot N_{\mathrm{s}}$ is the number of stiffeners. In this situation, the whole potential energy of all the system can be shown as:

$\Pi=U_{\mathrm{p}}+V_{\mathrm{p}}+U_{\mathrm{s}}+V_{\mathrm{s}}$

It is presupposed that rectangular plates are compressed uniformly in the direction of length, and the length and width of plates are $a$ and $b$ respectively. The instability mode of plates can be $m$ semi-waves in longitudinal direction and $n$ semi-waves in transverse direction. And in the case of situation that each side is fixed, plates buckling should satisfy these boundary situations:

When $x=0, a, w=0, \frac{\partial w}{\partial x}=0$,

When $y=0, b, w=0, \frac{\partial w}{\partial y}=0$. 
Buckling deflection function in correspond with boundary situations can be presupposed:

$$
w_{f}=f\left(1-\cos \frac{2 m \pi x}{a}\right)\left(1-\cos \frac{2 n \pi y}{b}\right)
$$

By substitution of buckling displacement function (6) into Eq. (1) to Eq. (5), after finishing arithmetic expressions, total potential energy $\Pi$ can be obtained.

A semi-wave of sheets, namely $m=n=1$, is studied in the longitudinal or transverse direction. Critical buckling stresses $\sigma$ are determined according to stationary principle of potential energy. And based on $\frac{\mathrm{d} \Pi}{\mathrm{d} f}=0, f \neq 0$, some equalities can be specified:

When $n_{s}=1$,

$$
\sigma=\frac{4\left(3 D b^{4}+3 D a^{4}+2 D a^{2} b^{2}+E I_{\mathrm{S}} b^{3}\right) t}{a^{2} b\left(3 b t+8 b_{\mathrm{s}} t_{\mathrm{S}}\right)}
$$

$$
\text { When } n_{s}>1 \text {, if } \frac{\pi}{\mathrm{n}_{\mathrm{s}}+1}=\chi
$$

$\sigma=\left(4 \pi^{2}\left(-3 E I_{S} b^{3} n_{s}+12 E I_{S} b^{3} \cos \chi^{2}-12 E I_{S} b^{3} \cos \chi^{4}+\right.\right.$

$+4 E I_{S} b^{3} \cos \chi^{6}+E I_{S} b^{3} \sin \chi^{2}+2 D a^{2} b^{2} \cos \chi^{2}-3 D a^{4}-$

$-3 D b^{4}-4 E I_{S} b^{3}+3 E I_{S} b^{3} n s \cos \chi^{2}-8 E I_{S} b^{3} \sin \chi^{2} \cos \chi^{2}+$

$+4 E I_{S} b^{3} \sin \chi^{2} \cos \chi^{4}-2 D a^{2} b^{2}+3 D a^{4} \cos \chi^{2}+$

$\left.\left.+3 D b^{4} \cos \chi^{2}\right)\right) /\left(\left(a^{2} b^{3}\left(-3 b_{s} t_{s} n_{s}+12 b_{s} t_{s} \cos \chi^{2}-\right.\right.\right.$

$-12 b_{s} t_{s} \cos \chi^{4}+4 b_{s} t_{s} \cos \chi^{6}+b_{s} t_{s} \sin \chi^{2}+$

$+4 b_{s} t_{s} \sin \chi^{2} \cos \chi^{2}+3 b_{s} t_{s} n_{s} \cos \chi^{2}-$

$\left.\left.-8 b_{s} t_{s} \sin \chi^{2} \cos \chi^{2}-4 b_{s} t_{s}+3 b t \cos \chi^{2}-3 b t\right)\right)$

Given that $\beta=\frac{a}{b}, \quad \delta=\frac{b_{\mathrm{s}} t_{\mathrm{s}}}{b t}, \quad \gamma=\frac{E I_{\mathrm{s}}}{D b}, \quad$ in above equalities, $\beta$ is length-width ratio of sheets, $\delta$ is area ratio between stiffeners and sheets, $\gamma$ is flexural rigidity ratio between stiffeners and sheets. Buckling coefficient $k$ can be obtained by Eq. (8):

$\sigma=k \frac{\pi^{2} D}{b^{2} t}$

When $n_{s}=1$, and if

$k=\frac{4\left(3+3 \beta^{4}+2 \beta^{2}+8 \gamma\right)}{\beta^{2}(3+8 \delta)}$

For CFRST columns without stiffeners under axial compression, namely $\beta=1, \delta=0, \gamma=0$, buckling coefficient $k$ is 10.67 , which is in accord with values in literature $[4,5]$ and verifies the correctness of deducing process in this paper. When length-width ratio $\beta$ is constant, buckling coefficients of stiffeners are presupposed to decrease with increasing area ratio $\delta$ and increase with increasing stiffener stiffness $\gamma$.
When $n_{s}>1$, if $\frac{\pi}{n_{\mathrm{s}}+1}=\chi$,

$k=\left(4\left(-3-2 \beta^{2}-4 \gamma+2 \beta^{2} \cos \chi^{2}-3 \gamma n_{\mathrm{s}}+\right.\right.$

$+12 \gamma \cos \chi^{2}-12 \gamma \cos \chi^{4}+3 \gamma n_{\mathrm{s}} \cos \chi^{2}-$

$-8 \gamma \sin \chi^{2} \cos \chi^{2}+4 \gamma \sin \chi^{2} \cos \chi^{4}+3 \cos \chi^{2}+$

$\left.\left.+3 \beta^{4} \cos \chi^{2}\right)\right) /\left(\left(\beta^{2}\left(3 \delta n_{\mathrm{s}} \cos \chi^{2}-\right.\right.\right.$

$-8 \delta \cos \chi^{2} \sin \chi^{2}+4 \delta \cos \chi^{4} \sin \chi^{2}-4 \delta+$

$+3 \cos \chi^{2}+3 \delta n_{\mathrm{s}}+\delta \sin \chi^{2}+12 \delta \cos \chi^{2}-$

$\left.\left.-12 \delta \cos \chi^{4}+4 \delta \cos \chi^{6}-3\right)\right)$

\section{DESIGN OF CFRST COLUMN STEEL TUBES WITH LONGITUDINAL STIFFENERS UNDER AXIAL COMPRESSION AND STIFFENERS}

\subsection{Limited Values of Width-Thickness about External Steel Tubes}

In order to make full use of strength of steel and decrease steel consumption, the thickness of sheets is relatively small. However, the smaller thickness is, the lower buckling strength is. For preventing sheet from locally elastically buckling, width-thickness ratio of external steel tubes about CFRST members is constrained [11]. The usual design criteria are elaborated as follows [12]:

(1) Critical stresses about local buckling of sheets are limited to higher than buckling strength, namely $\sigma_{c y} \geq f_{y}$.

(2) Critical stresses about local buckling of sheets are limited to higher than stresses about global instability of members, namely $\sigma_{c r} \geq \varphi_{f y}$.

(3) Critical stresses about local buckling of sheets are limited to higher than work stresses, namely $\sigma_{c r} \geq \sigma_{y}$.

The trends of critical stresses in criterion (1) to (3) are decreasing in order, as well as the margin of bearing capacity about plates design. Criterion (1) is an absolute stability, which is the most stringent design principle. And it is applied in Europe and America currently [13, 14]. Criterion (2) is equivalent stability principle, which is applied widely in China [15]. Based on criterion (1), this paper gives limited values of width-thickness ratios about CFRST columns with longitudinal stiffeners under axial compression.

In accordance with criterion (1), limited values can be determined. In other words, elastic buckling critical stresses of sheets are not allowed lower than buckling strength of steel.

$$
\sigma=k \frac{\pi^{2} D}{b^{2} t}=\frac{k}{12\left(1-v^{2}\right)} \frac{\pi^{2} E}{(b / t)^{2}} \geq f_{\mathrm{y}}
$$

Arithmetic Eq. (12) is finished:

$$
\frac{b}{t} \sqrt{\frac{f_{y}}{235}} \leq \sqrt{\frac{k \pi^{2} E}{12\left(1-v^{2}\right)} \cdot \frac{1}{235}}
$$


In Eq. (13), $\frac{b}{t} \sqrt{\frac{f}{235}}$ is relative width-thickness ratio of sheets. Based on this expression, the limited values of relative width-thickness ratio are determined by buckling coefficient. Take CFRST members with single stiffeners as an example, for CFRST columns under axial compression without stiffeners, $\delta=0, \gamma=0$, buckling coefficient $k$ equals 10.67. Theoretic limited values of relative widththickness about CFRST members under axial compression are 92 from expression 18. Considering some factors such as initial geometric imperfections of steel plates and welding residual stresses etc., it should reduce theoretic limited values [17]. From "CFRST structure technical regulation", recommended relative width-thickness limited value is 60. For CFRST columns with single stiffeners under axial compression, if stiffener stiffness is big enough, corresponding to providing clamped boundaries for plates near the stiffeners, plates will be split into two sub-elements, whose width becomes $b / 2$. Under axial compression loading, each sub-element buckles respectively. The buckling stress can be shown as:

$\sigma=10.67 \frac{\pi^{2} D}{(b / 2)^{2} t}=42.68 \frac{\pi^{2} D}{b^{2} t}$

From Eq. (14), ultimate buckle coefficient of CFRST columns with longitudinal stiffeners is 42.67, whose corresponding limited value of sub-element widththickness is 92. Considering the influences of some factors such as initial geometric imperfections of steel plates and welding residual stresses etc., this paper recommends that the limited value relative width-thickness ratio subelements of CFRST members with longitudinal stiffeners under axial compression is 60 .

\subsection{Design of Longitudinal Stiffeners}

As Fig. 4 shows the flexural rigidity ratio between stiffeners and sheets $\gamma$ determines buckling models of the sheets. When stiffener stiffness is not big enough, namely $\gamma<\gamma^{*}$, stiffeners will deform together with sheets under the loading, causing global stability of sheets. In that case, $\gamma^{*}$ is minimal stiffener stiffness ratio. If the sheets are globally stiffened, namely $\gamma>\gamma^{*}$, stiffeners will act as nodal lines.

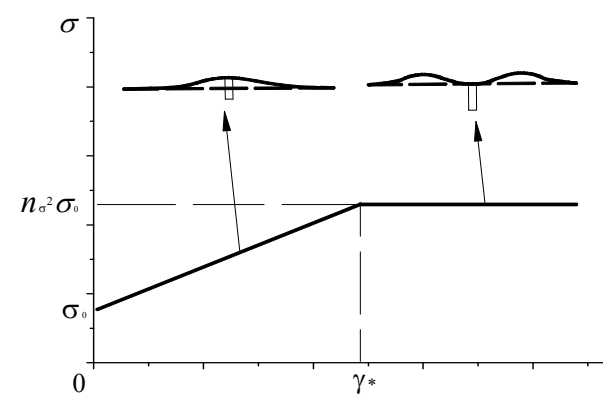

Figure 4 The relationship between flexural rigidity and buckling modes

Sub-elements among the stiffeners will buckle locally, whose local buckling critical stress will control the buckling stresses of sheets. In this condition, buckling stresses of sheets would not increase with flexural rigidity of stiffeners. Therefore, the primary thing about design of stiffeners is to determine the least flexural rigidity ratio of CFRST members under axial compression with longitudinal stiffeners.

\subsubsection{Member with a Single Stiffener}

First, the least flexural rigidity ratios can be determined. Take CFRST members under axial compression with single longitudinal stiffeners as an example in this paper. According to Eq. (10), if $\frac{\partial k}{\partial \beta}=0$, the minimum function $\mathrm{k}$ can be solved. In this condition, $\beta_{0}=\frac{1}{3}(81+216 \gamma)^{1 / 4}, \beta_{0}$ is a critical ratio between length and width of plates. By substitution of $\beta_{0}$ into Eq. (10), Eq. (15) can be solved:

$\beta_{0}=\frac{12\left(6+16 \gamma+\frac{2}{3} \sqrt{9+24 \gamma}\right)}{\sqrt{9+24 \gamma}(3+8 \delta)}$

When $\beta \leq \beta_{0}$, during the procession of the calculation of minimal flexural rigidity, the situation that sub-elements among stiffeners in transversal and longitudinal direction can form a semi-wave deformation is assumed. That is to say, buckling stresses of sub-elements $\sigma_{c r p}$ equal buckling stresses of sheets $\sigma$. On the simultaneous these two equations, Eq. (17) can be obtained:

$\sigma=k \frac{\pi^{2} D}{b^{2} t}, \sigma_{\mathrm{crp}}=k_{\mathrm{p}} \frac{\pi^{2} D}{(b / 2)^{2} t}$

$k=\frac{4\left(3+3 \beta^{4}+2 \beta^{2}+8 \gamma\right)}{\beta^{2}(3+8 \delta)}=4 k_{\mathrm{p}}$

Known buckling coefficients $k_{p}$ of CFRST columns under axial compression equal 10.67 . by substitution of $k_{p}$ into Eq. (17), the least flexural rigidity ratios can be determined:

$\gamma^{*}=-0.375+3.75 \beta^{2}+10.67 \beta^{2} \delta-0.375 \beta^{4}$

If $\beta>\beta_{0}$, the calculation of least flexural rigidity ratio is a simplification based on a semi-wave instability mode shape functions in transversal and longitudinal direction. Presuppose that $\beta=\beta_{0}$, then:

$k_{\min }=\frac{12\left(6+16 \gamma+\frac{2}{3} \sqrt{9+24 \gamma}\right)}{\sqrt{9+24 \gamma}(3+8 \delta)}=4 k_{\mathrm{p}}$

If $k_{p}=10.67$ is substituted into Eq. (19), then the least flexural rigidity ratios can be obtained: 


$$
\gamma^{*}=9+53.4 \delta+75.9 \delta^{2}
$$

In conclusion, the calculation formulas of least flexural rigidity ratio can be shown as follows:

$$
\gamma^{*}=\left\{\begin{array}{cc}
-0.375+3.75 \beta^{2}+10.67 \beta^{2} \delta-0.375 \beta^{4} & \beta \leq \beta_{0} \\
9+53.4 \delta+75.9 \delta^{2} & \beta>\beta_{0}
\end{array}\right.
$$

After reaching the minimal flexural rigidity ratios, presuppose that loading values when sheets are global instability equal bearing loads when sub-elements locally buckle. The buckling coefficients can be simplified as follows:

(1) If the length-width ratios of sheets are not bigger than critical ones, the coefficients can be straightly approximate calculation by shape functions.

(2) If the length-width ratios of sheets are bigger than critical ones, the adopted coefficients are the coefficients of minimum of a series of curves

\subsubsection{Member with Multiple Stiffeners}

As for CFRST columns with multiple stiffeners under axial compression, namely $n_{s}>1$, based on Eq. (11), if $\frac{\partial k}{\partial \beta}=0$, the minimum function of $k$ can be obtained. In this condition, $\beta_{0}=\left(\gamma+1+\gamma n_{\mathrm{s}}\right)^{1 / 4}$. By substitution of $\beta_{0}$ into expression 11, the function of $k_{\min }$ can be obtained.

If $\beta \leq \beta_{0}$, in the calculation of minimal flexural rigidity a hypothesis that sub-elements among the stiffeners form a semi-wave deformation should be assumed in transversal and longitudinal direction. Namely sub-elements' buckling stresses $\sigma_{c r p}$ equal buckling stresses $\sigma$. After simultaneous, k can be obtained:

$$
\begin{aligned}
& \sigma=k \frac{\pi^{2} D}{b^{2} t} \sigma_{c r p}=k_{p} \frac{\pi^{2} D}{\left(b /\left(n_{s}+1\right)\right)^{2} t} \\
& k=k_{\mathrm{p}}\left(n_{\mathrm{s}}+1\right)^{2}
\end{aligned}
$$

Known CFRST columns buckling coefficients under axial compression $k_{p}$ is 10.67 , where by substitution into Eq. (22), the minimal flexural rigidity ratios can be obtained:

$$
\begin{aligned}
& \gamma^{*}=\left(0 . 0 0 2 5 \left(1200+1200 M^{2}+1200 \beta^{4}-2400 \beta^{2}-3200 \beta^{2} n_{\mathrm{s}}{ }^{3} \delta-11700 \beta^{2} n_{\mathrm{s}} \delta-6400 \beta^{2} n_{\mathrm{s}}-3200 \beta^{2} n_{\mathrm{s}}{ }^{2}-\right.\right. \\
& -4270 \beta^{2} \delta+1070 \beta^{2} n_{\mathrm{s}}^{2} \delta N^{2}+3200 \beta^{2} n_{\mathrm{s}}^{3} \delta M^{2}+19200 \beta^{2} n_{\mathrm{s}}^{2} \delta M^{2}-12800 \beta^{2} n_{\mathrm{s}}{ }^{2} \delta M^{4}+4270 \beta^{2} n_{\mathrm{s}}{ }^{2} \delta M^{6}+ \\
& +2130 \beta^{2} n_{\mathrm{s}} \delta M^{2}-25600 \beta^{2} n_{\mathrm{s}} M^{4}+8540 \beta^{2} n_{\mathrm{s}} \delta M^{6}+28800 \beta^{2} n_{\mathrm{s}} \delta M^{2}-8540 \beta^{2} \delta N^{2} M^{2}+4270 \beta^{2} \delta N^{2} M^{4}- \\
& -10700 \beta^{2} n_{\mathrm{s}}^{2} \delta 3200 \beta^{2} n_{\mathrm{s}}{ }^{2} M^{2}+6400 \beta^{2} n_{\mathrm{s}} M^{2}+1070 \beta^{2} \delta N^{2}+12800 \beta^{2} \delta M^{2}-12800 \beta^{2} \delta M^{4}+4270 \beta^{2} \delta M^{6}+ \\
& \left.\left.+2400 \beta^{2} M^{2}-1200 \beta^{4} M^{2}-8540 \beta^{2} n_{\mathrm{s}}{ }^{2} \delta N^{2} M^{2}+4270 \beta^{2} n_{\mathrm{s}}{ }^{2} \delta N^{2} M^{4}-17100 \beta^{2} n_{\mathrm{s}} \delta N^{2} M^{2}+8540 \beta^{2} n_{\mathrm{s}} \delta N^{2} M^{4}\right)\right) \\
& /\left(-4-3 n_{\mathrm{s}}+12 M^{2}-12 M^{4}+4 M^{6}+N^{2}+3 n_{\mathrm{s}} M^{2}-8 N^{2} M^{2}+4 N^{2} M^{4}\right)
\end{aligned}
$$

When $\beta>\beta_{0}$, the calculation of minimal flexural rigidity ratios is based on a simplification of shape function of semi-wave instability modes in transversal and longitudinal direction. Presuppose that $\beta=\beta_{0}$, then:

$$
k_{\min }=\left(n_{\mathrm{s}}+1\right)^{2} k_{\mathrm{p}}
$$

By the substitution of $k_{p}=10.67$ into Eq. (24), the minimal flexural rigidity ratios can be obtained:

$$
\begin{aligned}
& \gamma^{*}=\frac{\pi}{n_{\mathrm{s}}+1}\left(1 . 7 \left(0.0048000+3.1 n_{\mathrm{s}}+1.5 \delta+8.7 \delta n_{\mathrm{s}}+\right.\right. \\
& +5.6 n_{\mathrm{s}}{ }^{2}+19 \delta n_{\mathrm{s}}{ }^{2}+20 \delta n_{\mathrm{s}}{ }^{3}+4.1 n_{\mathrm{s}}{ }^{3}+10 \delta n_{\mathrm{s}}{ }^{4}+n_{\mathrm{s}}{ }^{4}+ \\
& +\delta^{2}+15 \delta^{2} n_{\mathrm{s}}{ }^{2}+6.1 \delta^{2} n_{\mathrm{s}}+20 \delta^{2} n_{\mathrm{s}}{ }^{3}+15 \delta^{2} n_{\mathrm{s}}{ }^{4}+ \\
& \left.\left.+6.1 \delta^{2} n_{\mathrm{s}}{ }^{5}+\delta^{2} n_{\mathrm{s}}{ }^{6}+2 \delta n_{\mathrm{s}}{ }^{5}\right)\right)
\end{aligned}
$$

According to Eq. (26), common minimal flexural rigidity ratios of CFRST columns under axial compression with multiple stiffeners are listed in this paper, as shown in Tab. 1.

Table 1 the list of calculation expression about minimal flexural rigidity ratios
\begin{tabular}{|c|c|c|c|}
\hline $\begin{array}{c}\text { Rib } \\
\text { numbers }\end{array}$ & $\beta_{0}$ & $\beta \leq \beta_{0}$ & $\beta>\beta_{0}$ \\
\hline 2 & $(1+3 \gamma)^{1 / 4}$ & $24 \beta^{2} \delta+7.8 \beta^{2}-0.33-0.33 \beta^{4}$ & $45-280 \delta+430 \delta^{2}$ \\
\hline 3 & $(1+4 \gamma)^{1 / 4}$ & $43 \beta^{2} \delta+11 \beta^{2}-0.25-0.25 \beta^{4}$ & $110-900 \delta+1800 \delta^{2}$ \\
\hline 4 & $(1+5 \gamma)^{1 / 4}$ & $67 \beta^{2} \delta+13 \beta^{2}-0.2-0.2 \beta^{4}$ & $220+2200 \delta+5600 \delta^{2}$ \\
\hline 5 & $(1+6 \gamma)^{1 / 4}$ & $96 \beta^{2} \delta+16 \beta^{2}-0.17-0.17 \beta^{4}$ & $380+4600 \delta+14000 \delta^{2}$ \\
\hline 6 & $(1+7 \gamma)^{1 / 4}$ & $130 \beta^{2} \delta+19 \beta^{2}-0.14-0.14 \beta^{4}$ & $600+8500 \delta+30000 \delta^{2}$ \\
\hline
\end{tabular}

\section{CONCLUSION}

(1) The buckling analysis of CFRST columns under axial compression with longitudinal stiffeners can be simplified as solving the problem of the buckling loading of sheets with stiffeners under distributed loading, whose boundary conditions are sheets with clamped boundaries. What is more, the sheets can only deform on one side. Based on energy method, an analytical algorithm whose reliability can be proved about analyzing the buckling coefficients of CFRST columns under axial compression with longitudinal stiffeners is put forward in this paper.

(2) The minimal flexural rigidity ratios are the foundation of design about CFRST stiffeners. When the flexural rigidity is lacking, namely smaller than the 
minimal ratios, under the axial loading, stiffeners would deform together with sheets, developing global instability of sheets. If sheets are completely stiffening namely reaching the minimal ratios, stiffeners will act as nodal lines. Local buckling critical stresses of sub-elements among stiffeners would have control action. In this condition, buckling stresses of sheets would not continually increase with flexural rigidity of stiffeners.

(3) This paper determines the calculation formulas about solving minimal flexural rigidity ratios of steel tubes outside the CFRST members under axial compression with single or multiple stiffeners, putting forward limited values of relative width-thickness ratio among the stiffeners in steel tubes, providing profound evidence with the design of stiffeners such as the amount of ribs, cross section sizes and material etc.

\section{Acknowledgments}

This paper is supported by the National Natural Science Foundation of China (Contract No. 51778058), Natural Science Foundation of Shaanxi Provincial Department of Science and Technology (2018JQ5219), Natural Science Foundation of Qinghai Provincial Department of Transport (2017-ZI 13), Natural Science Foundation of Shaanxi Provincial Department of Transport (2017-30T).

\section{REFERENCES}

[1] Han, L., Tao, Z., \& Wang, W. (2009). Modern composite structure and mixed structure-experiment, theory and method. Beijing: science Press.

[2] Wright, H. D. (1995). Local Stability of Filled and Encased Steel Sections. Journal of Structural Engineering., 121(10), 1382-1388 https://doi.org/10.1061/(ASCE)0733-9445(1995)121:10(1382)

[3] Wright, H. D. (1993). Buckling of plates in contact with a rigid medium. The Structural Engineering., 71(12), 209-215.

[4] He, B., Yang, X., \& Zhou, T. (2002). Analytical analysis of local buckling behavior of rectangular concrete-filled steel tubular columns under axial compression. Journal of Xi'an University of Architectural Science and Technology (Natural Science Edition), 34(3), 210-213.

[5] Yang, X. (2002). Study on Local buckling behavior of concrete filled rectangular Steel Tubular columns. Xi'an, Xi'an University of Architectural Science and Technology.

[6] Mo, S., Zhong, X., \& Zhao, R. (2005). Buckling behavior of Elastic constrained rectangular plates on rigid substrates. Engineering mechanics, 22(2), 174-178.

[7] Cai, J., He, Z., \& Jin, X. (2007). Local buckling behavior of concrete-filled square steel tubular columns with restrained bars. Engineering mechanics, 24(5), 169-175.

[8] Cai, J. \& Long, Y. (2009). Local buckling of steel plates in rectangular CFT columns with binding bars. Journal of Constructional Steel Research, 65(4): 96972. https://doi.org/10.1016/j.jcsr.2008.07.025

[9] Cheng, R., Wang, Z., \& Shixiao, R. (2008). Application of stiffened ribbed ribs in concrete filled steel tube columns with large widths and thicknesses for example. Industrial buildings, 38(1), 100-102.

[10] Fu, B. (2004). Energy principle in elasticity and its Application. Beijing: science Press.

[11] Chen, S. (2004). Guide for stability design of steel structures. Beijing: China Construction Industry Press.
[12] Chen, S. (2009). Unified analysis of the limit value of width to thickness ratio of axial compression bar plate. Progress in building steel structures, (05), 1-7.

[13] Eurocode 4 (EC4) . (1994-1-1:2004). Design of steel and concrete structure-part1-1: General rules and rules for building. EN 1994-1-1:2004. Brussels : European committee for standardization.

[14] Aij. (2008). Recommendations for design and construction of concrete filled steel tubular structures.

[15] Ministry of Construction of the PRC. (2003). Code for design of steel structure : GB50017-2003. Beijing: China Planning Publishing House.

[16] Standards of China Association for Standardization of Engineering Construction. (2004). Technical specification for concrete filled rectangular tubular structures: CECS 159: 2004. Beijing: China Planning Press.

[17] Peng, K., Liu, Z., Zou, Q., Wu, Q., \& Zhou, J. (2020). Mechanical property of granite from different buried depths under uniaxial compression and dynamic impact: An energybased investigation. Powder Technology, 362, 729-744. https://doi.org/10.1016/j.powtec.2019.11.101

\section{Contact information:}

Hongping LIU, Senior Engineer

(Corresponding author)

Civil engineering design academy of Chang'an University Co., LTD, Chang'an University,

Middle-section of Nan'er Huan Road Xi'an, ShaanXi Province, 710064, China

E-mail: liuhongping_cau@163.com

Zhiheng ZHANG, Graduate Student

School of highway, Chang'an University,

Middle-section of Nan'er Huan Road Xi'an, ShaanXi Province, 710064, China

E-mail: Zhangzhiheng_edu@163.com

Zhang CHENG, Graduate Student

School of highway, Chang'an University,

Middle-section of Nan'er Huan Road Xi'an, ShaanXi Province, 710064, China E-mail:878453451@qq.com

Jin WEI, Professor

School of highway, Chang'an University,

Middle-section of Nan'er Huan Road Xi'an, ShaanXi Province, 710064, China E-mail:Weijin1972@163.com

Zeng GUOLIANG, Senior Engineer

Hunan Lianzhi Technology Co., Ltd,Changsha, Hunan,China,

Yanhe Road, Wangcheng Economic and Technological Development Zone, Changsha, Hunan Province,410000,China

E-mail:84893531@qq.com 